\title{
Glycan shield and epitope masking of a coronavirus spike protein observed by cryo-electron microscopy
}

\author{
Alexandra C Walls ${ }^{1}$, M Alejandra Tortorici ${ }^{2,3}$, Brandon Frenz ${ }^{1}$, Joost Snijder ${ }^{1}$, Wentao Li $^{4}$, Félix A Rey ${ }^{2,3}$, \\ Frank DiMaio ${ }^{1}$, Berend-Jan Bosch ${ }^{4} \&$ David Veesler ${ }^{1}$
}

The threat of a major coronavirus pandemic urges the development of strategies to combat these pathogens. Human coronavirus NL63 (HCoV-NL63) is an $\alpha$-coronavirus that can cause severe lower-respiratory-tract infections requiring hospitalization. We report here the 3.4-Å-resolution cryo-EM reconstruction of the HCoV-NL63 coronavirus spike glycoprotein trimer, which mediates entry into host cells and is the main target of neutralizing antibodies during infection. The map resolves the extensive glycan shield obstructing the protein surface and, in combination with mass spectrometry, provides a structural framework to understand the accessibility to antibodies. The structure reveals the complete architecture of the fusion machinery including the triggering loop and the $\mathrm{C}$-terminal domains, which contribute to anchoring the trimer to the viral membrane. Our data further suggest that HCoV-NL63 and other coronaviruses use molecular trickery, based on epitope masking with glycans and activating conformational changes, to evade the immune system of infected hosts.

Coronaviruses are enveloped viruses with large single-stranded positive-sense RNA genomes, classified in four genera $(\alpha, \beta, \gamma$ and $\delta)$. In humans, coronaviruses are responsible for $30 \%$ of respiratorytract infections ${ }^{1}$. In addition, coronaviruses have received substantial attention in the past decade, owing to the emergence of two deadly viruses with tremendous pandemic potential: severe acute respiratory syndrome coronavirus (SARS-CoV) and Middle East respiratory syndrome coronavirus (MERS-CoV) ${ }^{2}$. To date, there are no approved antiviral treatments or vaccines for any human coronavirus.

Coronaviruses are zoonotic viruses, and surveillance studies have suggested that both SARS-CoV and MERS-CoV originated from bats and that camels are also likely hosts for MERS-CoV ${ }^{3,4}$. Moreover, sequencing data have demonstrated that bats serve as a reservoir of coronaviruses that have the potential to cross the species barrier and infect humans. This phenomenon is illustrated by the observation that substitution of three amino acid residues in the spike (S) glycoprotein receptor-binding domain of the bat-infecting HKU4-CoV enhances its affinity for human DPP4 (the MERS-CoV receptor) by two orders of magnitude ${ }^{5,6}$. In addition, substitution of two other residues enables processing by human proteases and allows the HKU4-CoV S protein to mediate entry into human cells ${ }^{7}$. As a result, cross-species transmission of coronaviruses poses an imminent and long-term threat to human health. Recombination with coronaviruses frequently involved in mild respiratory infections may potentially lead to the emergence of highly pathogenic viruses ${ }^{4}$. Understanding the pathogenesis, cross-species transmission and recombination of coronaviruses is crucial to prevent or control their spread in humans and to evaluate the potential for long-term emerging diseases.
To date, $\alpha$ - and $\beta$-coronavirus genera have been implicated in human diseases and zoonoses. The human coronavirus NL63 (HCoV-NL63) is an $\alpha$-coronavirus that is genetically distinct from the $\beta$-coronaviruses mouse hepatitis virus (MHV, the prototypical coronavirus), MERS-CoV and SARS-CoV, and was first isolated from a 7 -month-old patient with a respiratory-tract infection ${ }^{8,9}$. Further studies have revealed that HCoV-NL63 infections appear to be common in childhood, and most adult sera contain antibodies that neutralize the virus ${ }^{8,10}$. HCoV-NL63 is a major cause of bronchiolitis and pneumonia in newborns worldwide and can cause severe lower-respiratory-tract infections that require hospitalization, especially among young children, the elderly and immunocompromised adults ${ }^{11}$. HCoV-NL63 infections have been reported in countries across Europe, Asia and North America, thus indicating its circulation among the human population worldwide. Other $\alpha$-coronaviruses related to the human respiratory pathogen $\mathrm{HCoV}-229 \mathrm{E}$ have recently been identified in camels co-infected with MERS-CoV${ }^{4}$, an observation further underscoring the importance of characterizing this coronavirus genus. Additionally, the emergence of the highly lethal porcine epidemic diarrhea coronavirus (PEDV, $\alpha$-genus) has recently had devastating consequences for the US swine industry ${ }^{12}$.

Coronaviruses use $\mathrm{S}$ homotrimers to promote cell attachment and fusion of the viral and host membranes. Because it is virtually the only antigen present at the virus surface, $S$ is the main target of neutralizing antibodies during infection and a focus of vaccine design ${ }^{13}$. S is a class I viral fusion protein that is synthesized as a single-chain precursor of $\sim 1,300$ amino acids and trimerizes after folding ${ }^{14}$. It is composed of 
an $\mathrm{N}$-terminal $\mathrm{S}_{1}$ subunit, containing the receptor-binding domain, and a C-terminal $S_{2}$ subunit, driving membrane fusion. After virion uptake by target host cells, cleavage at the $S_{2}{ }^{\prime}$ site (next to the putative fusion peptide) is required for fusion activation of all coronavirus $S$ proteins, so that they can subsequently transition to the postfusion conformation ${ }^{15-17}$.

Our previously reported cryo-EM reconstruction of the MHV S glycoprotein at 4.0 - $\AA$ resolution reveals the prefusion architecture of the machinery mediating entry of $\beta$-coronaviruses into cells ${ }^{18}$. It also demonstrates that coronavirus $S$ and paramyxovirus F proteins share a common evolutionary origin. Here, we set out to characterize the conservation of the $3 \mathrm{D}$ organization of spike proteins among coronaviruses belonging to different genera. We report the atomic-resolution structure of the pathogenic HCoV-NL63 $S$-glycoprotein trimer, which belongs to the $\alpha$-coronavirus genus. The substantial resolution improvement as compared with earlier studies allows visualization of the $S$ glycoprotein at an unprecedented level of detail, which is a prerequisite for guiding drug and vaccine design, and reveals both shared and unique features of the $\alpha$-genus of human pathogens. Our results suggest that HCoV-NL63 and other coronaviruses use molecular trickery, based on epitope masking with glycans and activating conformational changes, to evade the immune system of infected hosts, in a manner similar to that described for HIV-1.

\section{RESULTS}

\section{Structure determination}

We used Drosophila S2 cells to produce the HCoV-NL63 S ectodomain $\mathrm{N}$-terminally fused to a GCN4 trimerization motif downstream from the heptad-repeat 2 (HR2) helix. We imaged frozen-hydrated HCoVNL63 S ectodomain particles with an FEI Titan Krios electron microscope equipped with a Gatan Quantum GIF energy filter operated
Table 1 Data collection and refinement statistics

\begin{tabular}{lc}
\hline Data collection & 79,667 \\
Number of particles & 1.36 \\
Pixel size $(\AA)$ & $2-4$ \\
Defocus range $(\mu \mathrm{m})$ & 300 \\
Voltage $(\mathrm{kV})$ & 48 \\
Electron dose $\left(\mathrm{e}^{-} / \AA^{2}\right)$ & \\
Refinement & 3.4 \\
Resolution $(\AA)$ & -129 \\
Map-sharpening $B$ factor $\left(\AA^{2}\right)$ & \\
Model validation & 97.87 \\
Favored rotamers $(\%)$ & 0.68 \\
Poor rotamers $(\%)$ & 99.32 \\
Ramachandran allowed $(\%)$ & 0.6 \\
Ramachandran outliers $(\%)$ & 3.3 \\
Clash score & 1.54 \\
MolProbity score &
\end{tabular}

in zero-loss mode, with a slit width of $20 \mathrm{eV}$, and a Gatan K2 Summit electron-counting camera ${ }^{19}$ (Online Methods).

We determined a 3D reconstruction of the HCoV-NL63 S at 3.4-Å resolution, using the gold-standard Fourier shell correlation (FSC) criterion of 0.143 (refs. 20,21) (Fig. 1 and Supplementary Fig. 1). The final model, which we built and refined with $\operatorname{Coot}^{22}$ and Rosetta ${ }^{23-25}$, includes residues 23 to 1224, with internal breaks between residues 110-121, 882-890 and 992-1001 (Supplementary Fig. 1 and Table 1). The HCoV-NL63 S ectodomain is a 160- $\AA$-long trimer with a triangular cross-section.

\section{The ordered glycan shield}

A notable feature of this structure is the extraordinary number of $\mathrm{N}$-linked oligosaccharides that cover the spike trimer. In the cryo-EM

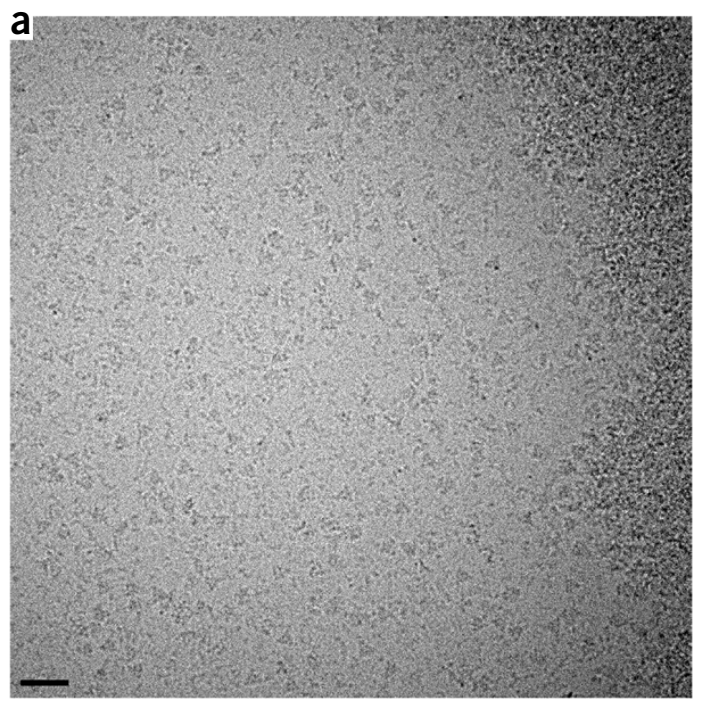

b

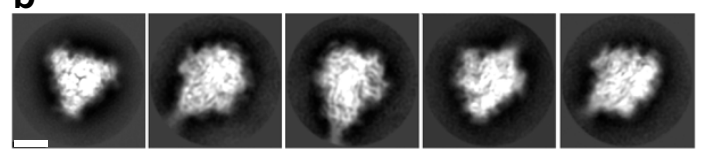

C
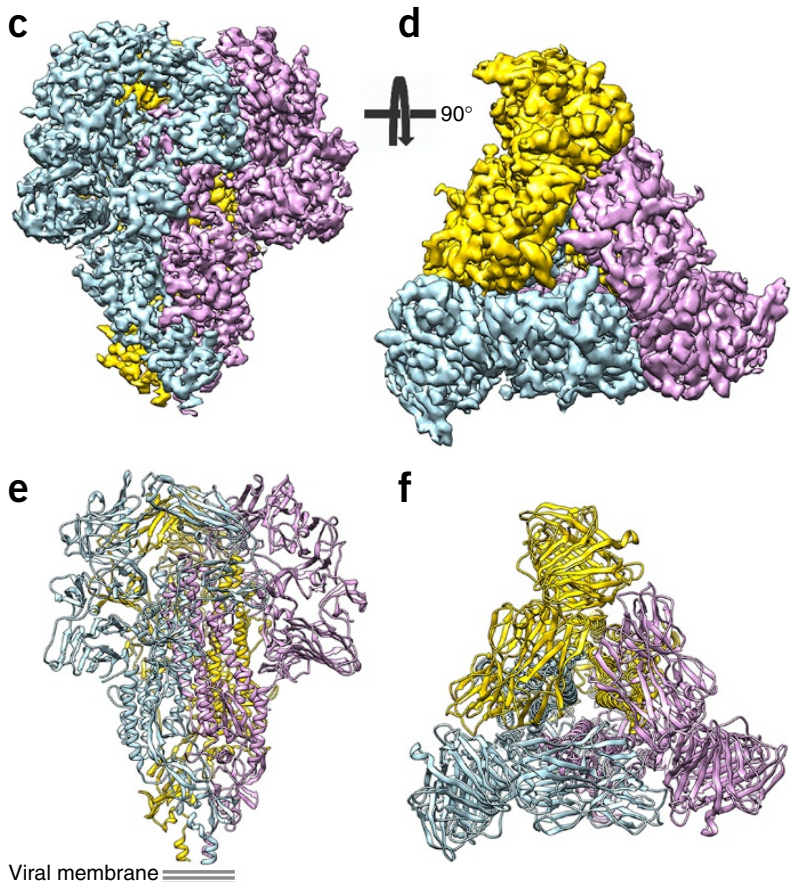

Figure 1 Cryo-EM structure of the HCoV-NL63 S trimer. (a) Representative micrograph of frozen-hydrated HCoV-NL63 S particles (defocus $3.4 \mu \mathrm{m}$ ). Scale bar, $355 \AA$. (b) Five selected class averages showing the particles along different orientations. Scale bar, $60 \AA$. (c,d) 3D map filtered at 3.4- $\AA$ resolution and colored by protomer. Two orthogonal views of the $\mathrm{S}$ trimer from the side (c) and from the top, facing toward the viral membrane, (d) are shown. (e,f) Ribbon diagrams showing the HCoV-NL63 S atomic model, oriented as in $\mathbf{c}$ and $\mathbf{d}$, respectively. 
a

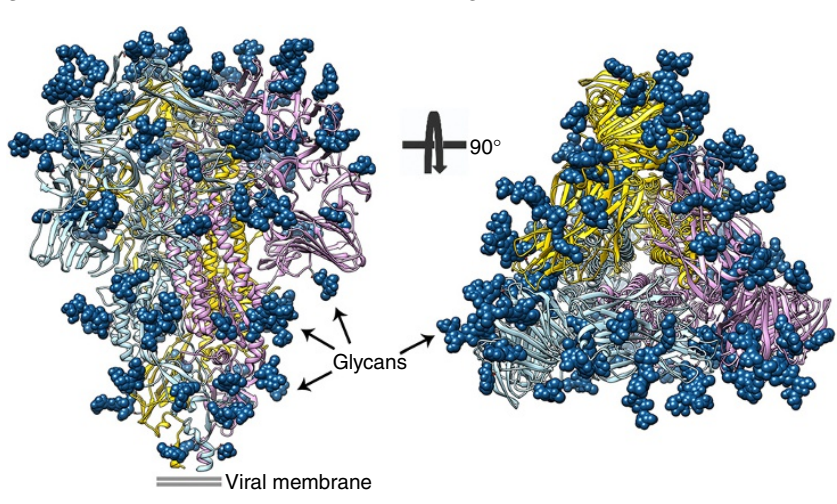

C

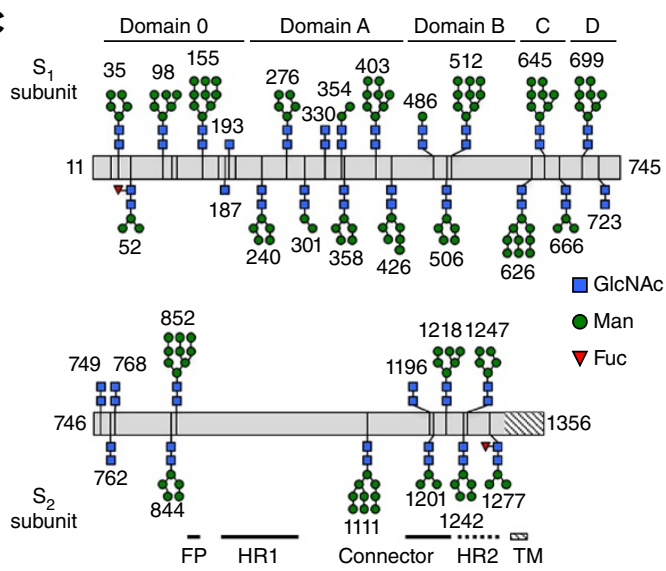

Figure 2 Organization of the HCoV-NL63 S-protein glycan shield, revealed by cryo-EM and MS. (a,b) Ribbon diagrams showing two orthogonal views of the $\mathrm{S}$ trimer, from the side (a) and from the top (b), facing toward the viral membrane. Glycans are shown as dark-blue spheres. (c) Residue-level schematic of $\mathrm{N}$-linked glycans. The most extensive glycan structure detected by MS at each site is represented except for glycans observed only by cryo-EM, for which the resolved sugar moieties are shown. FP, fusion peptide; HR1, heptad-repeat 1 region; HR2, heptad-repeat 2 region (shown with a dashed line because it is not resolved in the map); TM, transmembrane domain (the striated texture indicates regions that are not part of the construct); GlcNac, $N$-acetylglucosamine; Man, mannose; Fuc, fucose.

reconstruction, we observed density for 31 N-linked glycans extending tangentially relative to the protein surface (Fig. 2a,b, Supplementary Fig. 1 and Supplementary Table 1). At least the two core $\mathrm{N}$-acetylglucosamine moieties are visible for the majority of glycosylation sites.

Using on-line reversed-phase liquid chromatography with electron transfer/high-energy collision-dissociation tandem $\mathrm{MS}^{26}$, we detected $25 \mathrm{~N}$-linked glycosylation sites overlapping with those observed in the cryo-EM map and identified three additional sites (Fig. 2c, Supplementary Fig. 2 and Supplementary Table 1). We identified these sites from both intact glycopeptides and peptides with the glycan trimmed down to the $\mathrm{N}$-linked core $\mathrm{N}$-acetylglucosamine moiety. The cryo-EM and MS data together provide evidence for glycosylation at 34 out of 39 possible NXS/T glycosylation sequons. The intact glycopeptides detected by MS/MS for HCoV-NL63 S expressed in Drosophila S2 cells corresponded to either paucimannosidic glycans containing three mannose residues (with or without core fucosylation) or high-mannose glycans containing four to nine mannose residues. Although glycan processing differences exist between insect and mammalian cell expression systems, the same glycosylation sequons are expected to be recognized and glycosylated in both cases. Previous reports have suggested that several coronavirus S glycans are of the high-mannose type, as a result of direct budding from the endoplasmic reticulum-Golgi intermediate compartment ${ }^{27,28}$, thus supporting the biological relevance of the potential glycan structures identified.

In the refined model, $\mathrm{N}$-linked glycans cover a substantial amount of the accessible surface of the trimer (Fig. 2a,b). The higher glycan density per accessible surface area detected for the $S_{2}$ subunits (819 $\AA^{2} /$ glycan) compared with the $S_{1}$ subunits (1,393 $\AA^{2} /$ glycan) may explain why most coronavirus neutralizing antibodies isolated to date target the latter region. Because many of the observed glycosylation sites are topologically conserved among coronavirus S proteins, we suggest that the glycan footprint observed here may be representative of those of other S proteins. Besides potentially contributing to immune evasion, as discussed below, $\mathrm{S}$ glycans have been proposed to play a role in host-cell entry ${ }^{29}$ via L-SIGN lectin, which is an alternative receptor for SARS-CoV 30 and $\mathrm{HCoV}-229 \mathrm{E}^{27}$.

\section{Structure of the $S_{2}{ }^{\prime}$ trigger loop}

The HCoV-NL63 and MHV $S_{2}$ fusion machineries are structurally similar and can be superimposed with excellent agreement (Fig. 3a and Supplementary Fig. 3; DALI ${ }^{31} Z$ score 29.7, r.m.s. deviation $2.2 \AA ̊$ over 312 residues). In contrast to our previous MHV S structure ${ }^{18}$, most of the $\mathrm{HCoV}-\mathrm{NL} 63 \mathrm{~S}_{2}{ }^{\prime}$ trigger loop, which connects the upstream helix to the fusion peptide and participates in fusion activation, is resolved in the reconstruction (Fig. 3b). The trigger loop runs almost perpendicularly to the long axis of the $\mathrm{S}_{2}$ subunit and forms three helical segments before looping back to connect to the fusion peptide. Multiple arginine residues, forming two putative furin-cleavage sites, are present in the C-terminal region of the $\mathrm{S}_{2}{ }^{\prime}$ loop (863-RNIRSSR-870), which is characterized by weaker density, as would be expected from a proteasesensitive polypeptide segment. These observations are consistent with results of previous studies suggesting that fusion activation of the HCoV-NL63 S glycoprotein occurs after $\mathrm{S}_{2}{ }^{\prime}$ proteolytic processing at the plasma membrane (by trypsin-like proteases such as TMPRS2) or in the endosomal pathway (by furin or cysteine proteases) ${ }^{15,32}$.

The lack of strict amino acid sequence conservation at the $S_{2}{ }^{\prime}$ cleavage site among coronavirus $S$ proteins reflects the usage of different proteases found in distinct cellular compartments for fusion activation $^{15,17}$. Similarly to the additional cleavage site present between the $S_{1}$ and $S_{2}$ subunits of MERS-CoV ${ }^{7}$, the multiple glycans present in the vicinity of the $S_{2}^{\prime}$ loop probably further influence protease sensitivity (Fig. 3b). However, we emphasize that $S_{2}{ }^{\prime}$ processing occurs at topologically equivalent positions for $\mathrm{HCoV}$-NL63 S, MERS-CoV S, MHV S and probably most coronavirus S glycoproteins.

\section{Anchoring of the fusion machinery to the viral membrane}

The HCoV-NL63 S reconstruction (Fig. 3a) resolves a large part of the $\mathrm{S}_{2} \mathrm{C}$-terminal region that has not been observed in previous studies ${ }^{18,33}$. We were able to build an atomic model for the connector domain and the stem helix, which connect to the HR2 region. The connector folds as a $\beta$-rich domain decorated with one short $\alpha$-helix. At its C-terminal end, the polypeptide chain folds as an $\alpha$-helix (stem helix, Fig. 3a,c,d) aligned along the three-fold molecular axis, which turns into the HR2 domain, corresponding to 71 additional residues not resolved in our map. In the trimer, the connector domains 

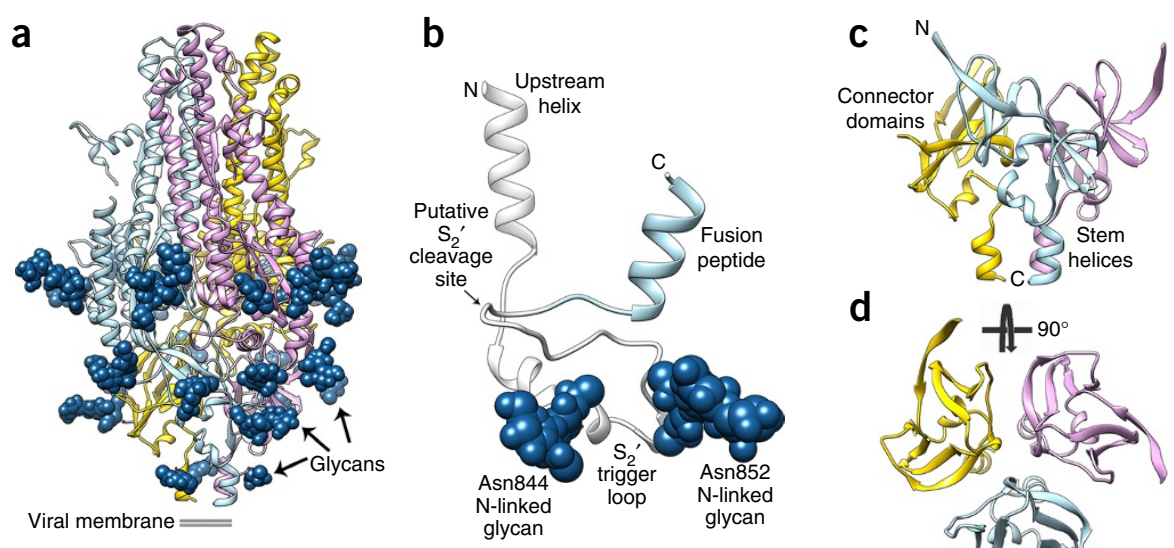

d
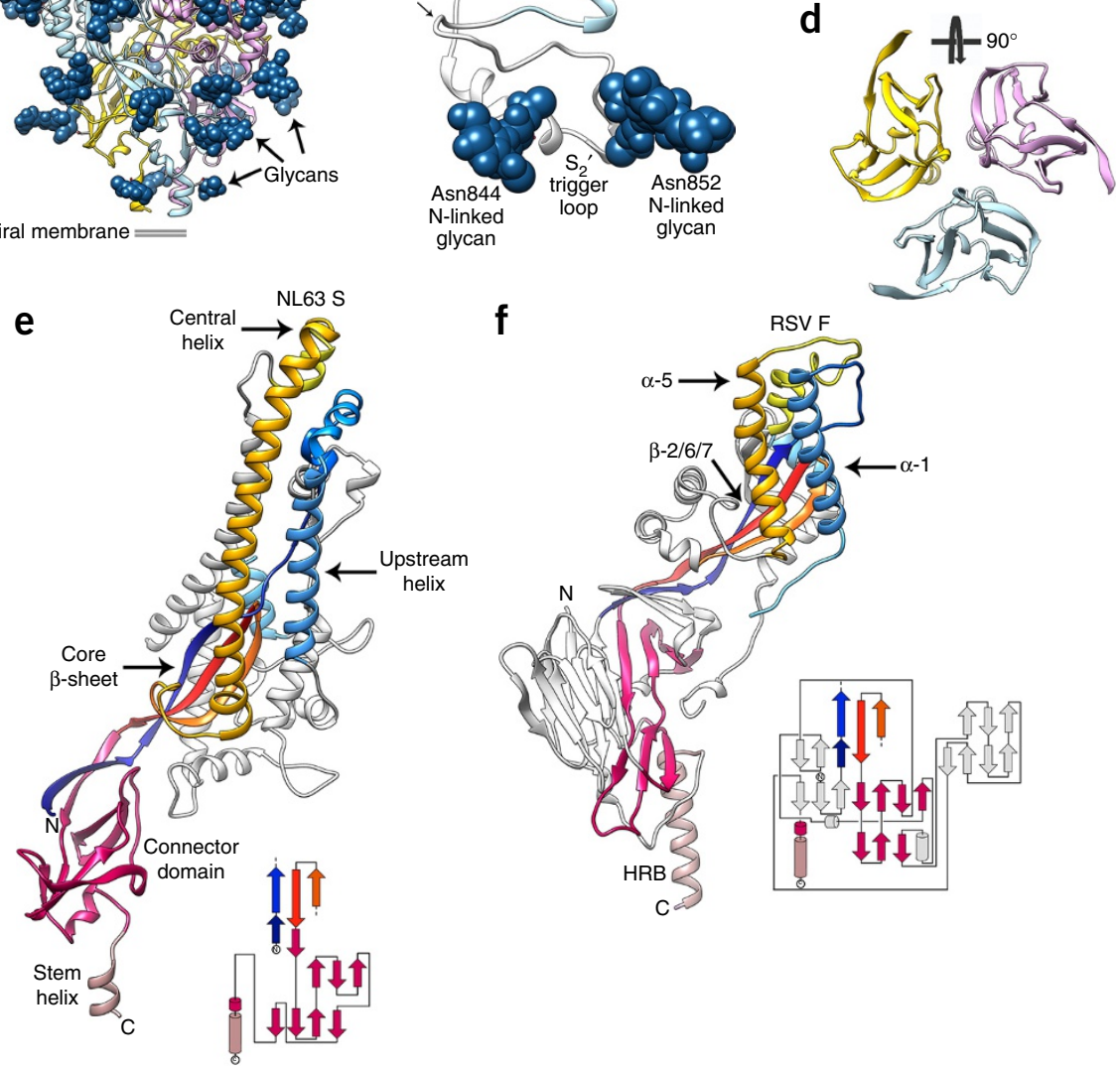

Figure 3 Architecture of the complete coronavirus fusion machinery. (a) Ribbon diagram of the $\mathrm{S}_{2}$ trimer, colored by protomer with glycans rendered as dark-blue spheres. (b) Zoomed-in view of the $\mathrm{S}_{2}{ }^{\prime}$ trigger-loop region comprising the central helix and the fusion peptide (light blue). $\mathrm{N}$-linked glycans are shown as dark-blue spheres. The polypeptide segment corresponding to the putative cleavage site is poorly resolved in the density, and this part of the model should be considered to be hypothetical. (c,d) Ribbon diagrams showing two orthogonal views of the $\mathrm{S}_{2}{ }^{\prime}$ C-terminal region, which is assembled from the connector domains and stem helices. (e,f) Ribbon diagrams of the HCoV-NL63 $\mathrm{S}_{2}$ subunit (e) and of the RSV F protein (f). Conserved structural elements are colored identically to highlight the similar 3D organization of these two fusion machineries, whereas nonconserved regions are colored gray. The topology diagrams underscore the similar topology of the HCoV-NL63 S connector domain and the equivalent RSV F domain, although the tertiary structures of these domains are different, and several structural motifs have been added to the latter domain throughout evolution. The RSV F secondary-structural elements are annotated according to ref. 34 . The $\mathrm{N}$ - and C-terminal extremities of the polypeptide segments are indicated.

assemble as a cup flanking the viral membrane-proximal side of the ectodomain, and the stem helices form a bundle stabilized by hydrophobic interactions.

The coronavirus $\mathrm{S}$ connector domain and the equivalent paramyxovirus $\mathrm{F}$ domain share a related topology, although their tertiary structures are different, and several structural motifs have been added to the latter domain throughout evolution ${ }^{34,35}$ (Fig. 3e,f). Moreover, the trimer of stem helices assembles as a helical bundle, which initiates the HR2 domain in a manner reminiscent of the heptad repeat $\mathrm{B}$ (HRB) region of paramyxovirus prefusion $\mathrm{F}$ structures ${ }^{34,35}$. These observations lend further support to the evolutionary connection that we have previously proposed for the fusion machineries of these two viral families ${ }^{18}$.

Comparison of the prefusion HCoV-NL63 $\mathrm{S}_{2}$ subunit with the structure of the postfusion core suggests that the $\mathrm{C}$-terminal region of the connector domain and the stem helix must refold and/or change conformation to yield the canonical 'trimer of hairpin' conformation that mediates fusion of the host and viral membrane in all class I fusion proteins ${ }^{18,36,37}$.

\section{Duplication of the $\mathrm{N}$-terminal domain in $\alpha$-coronaviruses}

The HCoV-NL63 S structure shows the presence of an additional $\mathrm{N}$-terminal domain not present in $\beta$-coronaviruses. Phylogenetic analyses suggest that this is a canonical feature of most $\alpha$-coronavirus $S$ glycoproteins (Fig. $\mathbf{4 a - c}$ ). This domain, which we named domain 0 , adopts a galectin-like $\beta$-sandwich fold supplemented with a three-stranded $\beta$-sheet, similarly to domain A (Fig. 4d-f, DALI $Z$ score 6.7, r.m.s. deviation 3.8 Å over 147 residues), thus suggesting a gene-duplication event. Domain 0 interacts with the viral-membraneproximal side of domain $\mathrm{A}$ and with domain $\mathrm{D}$. 
a

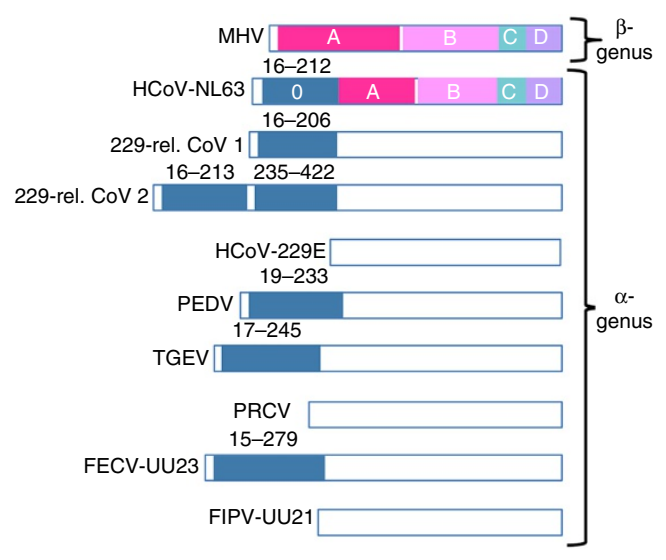

b
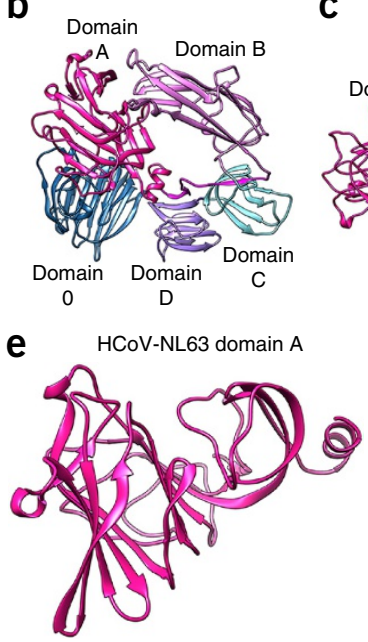

C

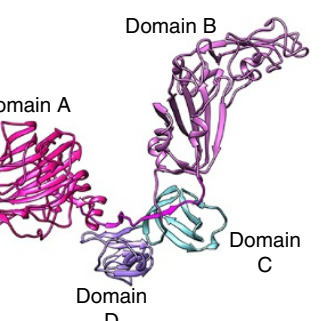

D

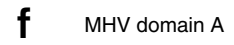

f MHV domain A

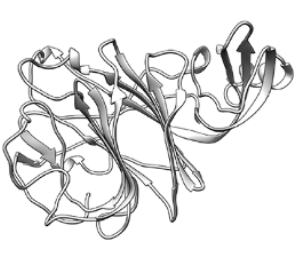

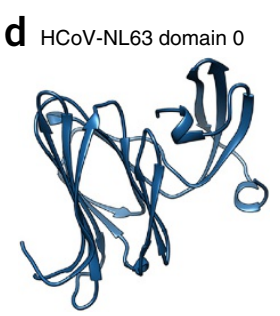

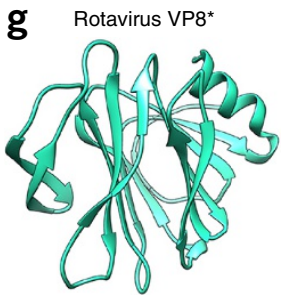

Figure 4 Evolution of the $\alpha$-coronavirus S-glycoprotein fold appears to correlate with tissue tropism. (a) Schematic representation of several $\alpha$-coronavirus S-glycoprotein S1 subunits, highlighting the presence of one or several domains 0 (blue), as compared with $\beta$-coronaviruses. HCoV-NL63 (GenBank YP_003767.1), 229-rel. CoV 1 (GenBank ALK28775.1), 229-rel. CoV 2 (GenBank ALK28765.1), HCoV-229E (GenBank NP_073551.1), porcine epidemic diarrhea virus (PEDV; GenBank AAK38656.1), transmissible gastroenteritis virus strain Purdue P115 (TGEV; GenBank ABG89325.1), porcine respiratory coronavirus strain ISU-1 (PRCV; GenBank ABG89317.1), feline enteric coronavirus strain UU23 (FECV-UU23; GenBank ADC35472.1) and feline infectious peritonitis coronavirus strain UU21 (FIPV-UU21; GenBank ADL71466.1). The $\beta$-coronavirus MHV $\mathrm{S}_{1}$ subunit is shown for comparison. Domains A-D are indicated for MHV and HCoV-NL63. (b) Ribbon diagram of the HCoV-NL63 $\mathrm{S}_{1}$ subunit. (c) Ribbon diagram

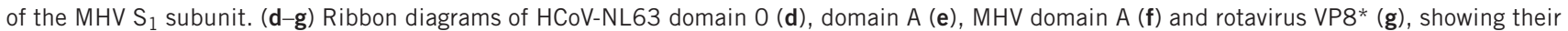
structural similarity, which suggests common ancestry. HCoV-NL63 domain 0 and A probably arose from a duplication event.

We determined that domain 0 is also structurally similar to the $\mathrm{VP}^{*}$ sialic acid-binding domain of the rotavirus VP4 spike protein ${ }^{38}$ (Fig. 4g; PDB 1KQR, DALI $Z$ score 8.9, r.m.s. deviation $3.0 \AA$ over 109 residues). In line with this finding, domain 0 of transmissible gastroenteritis coronavirus (TGEV) and of PEDV bind to sialic acid, and deletion of this domain in $\alpha$-coronavirus $S$ appears to correlate with a loss of enteric tropism ${ }^{39}$. We detected no sialic acid binding activity for the HCoV-NL63 $S_{1}$ subunit (Supplementary Fig. 4), thus possibly explaining the strict respiratory tropism of this virus. Instead, host-cell heparan sulfate proteoglycans have been shown to participate in HCoV-NL63 anchoring and infection ${ }^{40}$, and we detected binding of heparan sulfate to the HCoV-NL63 S protein by using surface plasmon resonance (SPR) (Supplementary Fig. 5a). We hypothesize that these interactions may be mediated either by domain 0 , which exhibits several positively charged patches on its surface (Supplementary Fig. 5b), or domain A, which has been reported to bind carbohydrates in the case of a bovine coronavirus ${ }^{41}$.

\section{A putative immune-evasion strategy}

Domain $\mathrm{B}$, which is the $\mathrm{HCoV}-\mathrm{NL} 63$ receptor-binding domain, exhibits a structure distinct from those of $\beta$-coronavirus B domains, although a topological relatedness has been detected among these $\beta$-rich domains ${ }^{42}$. Superimposition of the HCoV-NL63 and MHV $\mathrm{S}_{1}$ subunits highlights that their B domains feature opposite orientations related by an $\sim 180^{\circ}$ rotation (Fig. 5a,b). As a result, many of the $\mathrm{HCoV}-\mathrm{NL} 63$ receptor-binding residues are buried through interaction with domain $\mathrm{A}$ of the same protomer, are masked by the glycan at residue Asn358 and are not available to engage the host-cell receptor (human angiotensin-converting enzyme 2, ACE2). Comparison of the HCoV-NL63 domain-B structure in our cryo-EM-derived model with the crystal structure of the same domain in complex with ACE2 (ref. 43) revealed that the receptor-binding loop containing residues 531-539 undergoes substantial conformational changes after binding (and is defined by weak density; Fig. 5c). These findings explain the markedly higher ACE2 binding affinity of HCoV-NL63 domain B, compared with that of the full-length $S_{1}$ domain (Fig. 5d).

Because the receptor-binding loops elicit potent neutralizing antibodies in the case of $\mathrm{TGEV}^{44}$, MERS-CoV ${ }^{45}$ and SARS-CoV ${ }^{46-49}$, we speculate that HCoV-NL63 has evolved to limit exposure of this vulnerable site to $\mathrm{B}$-cell receptors via protein-protein interactions and glycan masking. This mechanism is reminiscent of the HIV-1 immune evasion strategy, which relies on a glycan shield and conformational changes that are triggered by binding of CD 4 and expose the chemokine-receptor-interacting motifs ${ }^{50,51}$.

\section{DISCUSSION}

Viruses have evolved several immune-evasion strategies including rapid antigenic evolution, masking of epitopes and exposure of nonneutralizing immune-dominant 'decoy' epitopes. For example, HIV-1 (ref. 52), Lassa virus ${ }^{53}$, hepatitis $C$ virus ${ }^{54}$ and Epstein-Barr virus ${ }^{55}$ exhibit extensive $\mathrm{N}$-linked glycosylation, covering exposed protein surfaces, with glycan masses that may exceed that of the protein component. The HCoV-NL63 S trimer is covered by an extensive glycan shield consisting of $102 \mathrm{~N}$-linked oligosaccharides obstructing the protein surface. This observation is reminiscent of descriptions of the HIV-1 envelope trimer ${ }^{52}$, although the glycan density is $30 \%$ higher in the latter case. Furthermore, our data suggest that, similarly to HIV-1, coronavirus S glycans mask the protein surface and consequently limit access to neutralizing antibodies and thwart the humoral immune response. This strategy is illustrated by the presence of a glycan linked to Asn358 in the HCoV-NL63 structure reported here. This glycan, along with the proteinaceous moiety of domain A, contributes to masking the receptor-binding loops, which have been shown to elicit potent neutralizing antibodies for other coronaviruses ${ }^{44-49}$ and appear to represent a potential 'Achilles' heel' of these viruses. This hypothesis is further supported by the observation of three additional glycans directly protruding from the viral-membranedistal side of domain B. As a result, conformational changes are 

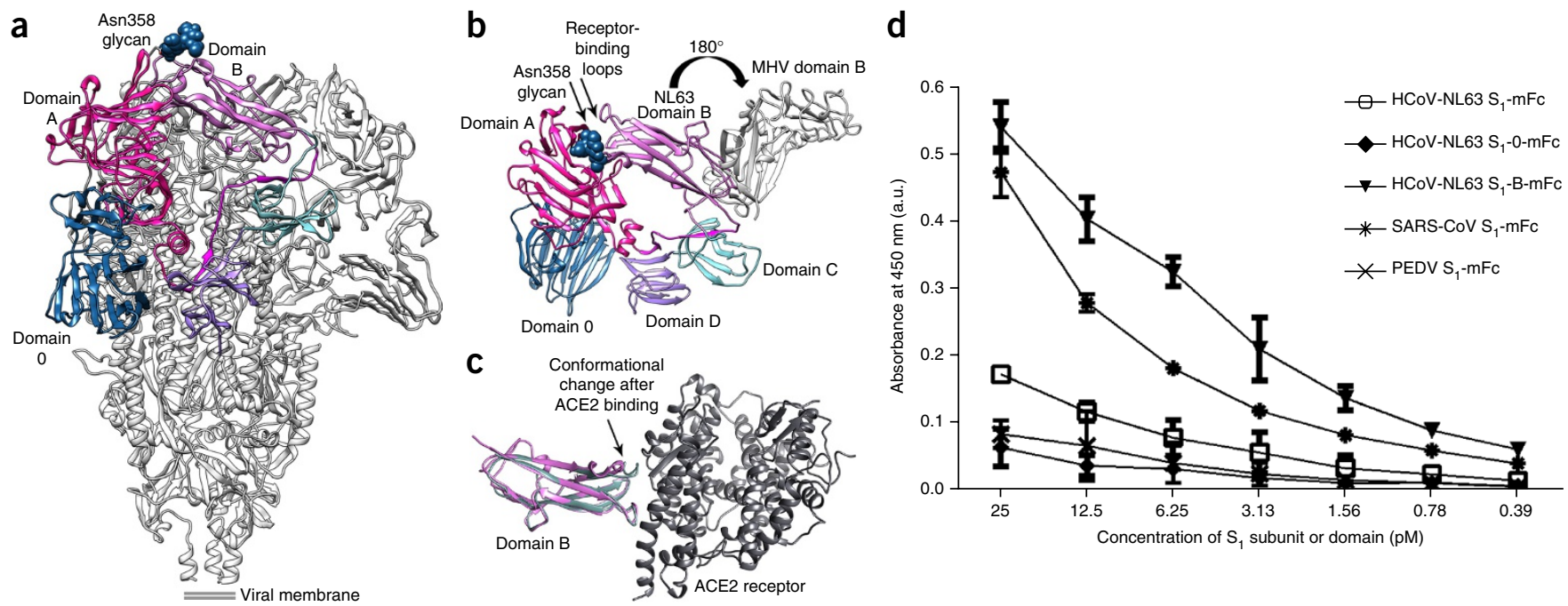

Figure 5 Potential immune-evasion strategy used by HCoV-NL63. (a) Ribbon diagram of the HCoV-NL63 S trimer, highlighting the conformation of the $\mathrm{S}_{1}$ subunit. Domains $\mathrm{O}, \mathrm{A}, \mathrm{B}, \mathrm{C}$ and $\mathrm{D}$ are colored for one protomer. (b) The HCoV-NL63 receptor-binding loops are buried via interactions with domain A of the same protomer (including the glycan moiety at Asn358) and are not available to engage host-cell receptors. Superimposition of the HCoV-NL63 (purple) and MHV (light gray) $\mathrm{S}_{1}$ subunits via their $\mathrm{C}$ domains highlights that their $\mathrm{B}$ domains feature opposite orientations related by an $\sim 180^{\circ}$ rotation, thus suggesting a putative trajectory for the conformational changes that must occur to engage the host-cell receptor. Only domain B is shown for MHV S. (c) Comparison of the HCoV-NL63 domain-B structure in our cryo-EM-derived model (purple) with the crystal structure of the same domain in complex with ACE2 (green and dark gray), showing that the receptor-binding loop containing residues 531-539 substantially changes its conformation after binding. (d) ACE2 binding ELISA showing that isolated HCoV-NL63 domain B (HCoV-NL63 $\mathrm{S}_{1}$-B-mFc) binds ACE2 with higher affinity than does the full-length $\mathrm{S}_{1}$ domain (HCoV-NL63 $\mathrm{S}_{1}-\mathrm{mFc}$ ). SARS-CoV $\mathrm{S}_{1}$ (HCoV-NL63 $\mathrm{S}_{1}-\mathrm{mFc}$ ) is a positive control. HCoV-NL63 $\mathrm{S}_{1}$ domain 0 (HCoV-NL63 $\mathrm{S}_{1}-0$-mFc) and PEDV $S_{1}\left(P E D V S_{1}-m F c\right)$, which do not bind ACE2, are negative controls. Mean values and s.d. of three independent experiments are shown.

required for the $\mathrm{HCoV}$-NL63 $\mathrm{S}$ glycoprotein to be able to interact with ACE2 (ref. 43). These rearrangements and/or receptor binding are likely to participate in initiating the fusion reaction by disrupting the interactions formed between domain B and the HR1 C-terminal region. Interactions with heparan sulfate proteoglycans present at the host-cell surface might potentially contribute to activating HCoV-NL63 S and promote subsequent interactions with ACE2. A common theme arising from the analysis of $\alpha$ - and $\beta$-coronavirus S-glycoprotein structures is that domain-B-mediated host anchoring involves major structural rearrangements that expose the binding motifs ${ }^{18,33}$.

Visualization of the glycan shield obstructing access to the S surface and deciphering the molecular trickery used by some coronaviruses provide a rational basis for understanding the accessibility to neutralizing antibodies and may pave the way for guiding future design of immunogens or therapeutics. We have previously suggested that targeting the fusion machinery bears the promise of finding broadly neutralizing inhibitors of coronavirus infection ${ }^{18}$, and the high density of glycans decorating this region will need to be taken into consideration to increase the likelihood of success.

\section{METHODS}

Methods and any associated references are available in the online version of the paper.

Accession codes. The cryo-EM map has been deposited in the Electron Microscopy Data Bank under accession code EMD-8331; the corresponding atomic model has been deposited into the Protein Data Bank under accession code PDB 5SZS. The MS data (including the raw data, COMET search results and annotated tandem MS spectra of all accepted glycopeptide identifications) have been deposited in the proteomics identifications (PRIDE) database under dataset PXD004557.
Note: Any Supplementary Information and Source Data files are available in the online version of the paper.

\section{ACKNOWLEDGMENTS}

Research reported in this publication was supported by the National Institute of General Medical Sciences (NIGMS) of the National Institutes of Health (NIH) under award number 1R01GM120553-01 (D.V.) and T32GM008268 (A.C.W.) J.S. acknowledges support from the Netherlands Organization for Scientific Research (NWO, Rubicon 019.2015.2.310.006) and the European Molecular Biology Organisation (EMBO, ALTF 933-2015). M.A.T. and F.A.R. acknowledge support from the Institute Pasteur and the CNRS. The authors acknowledge the use of instruments at the Electron Imaging Center for NanoMachines supported by the NIH (1S10RR23057 and 1S10OD018111), NSF (DBI-1338135) and CNSI at UCLA. The authors are grateful to H. Choe (The Scripps Research Institute) for providing the HCoV-NL63 S gene, J. Labonte (Johns Hopkins School of Medicine) for providing the Rosetta code handling glycans and P.J.M. Rottier (Utrecht University) for scientific advice. This work was partly supported by the University of Washington's Proteomics Resource (UWPR95794), and the authors thank P.D. von Haller and J.K. Eng for their assistance and expertise. SPR experiments were performed with the help of J. Sumida at the University of Washington School of Pharmacy Analytical Biopharmacy Core. Part of this research was facilitated by the Hyak supercomputer system at the University of Washington.

\section{AUTHOR CONTRIBUTIONS}

B.-J.B. designed and cloned the protein construct. M.A.T. carried out protein expression and purification under the supervision of F.A.R. A.C.W. performed cryo-EM sample preparation, data collection and processing under the supervision of D.V. A.C.W., B.F., F.D. and D.V. built the atomic model. A.C.W. and J.S. performed the MS experiments. W.L. performed the hemagglutination assays and ELISAs under the supervision of B.-J.B. A.C.W. performed the SPR experiments under the supervision of D.V. All authors analyzed the data. A.C.W., J.S., B.-J.B. and D.V. prepared the manuscript with input from all authors.

\section{COMPETING FINANCIAL INTERESTS}

The authors declare no competing financial interests.

Reprints and permissions information is available online at http://www.nature.com/ reprints/index.html. 
1. Zumla, A., Chan, J.F., Azhar, E.I., Hui, D.S. \& Yuen, K.Y. Coronaviruses: drug discovery and therapeutic options. Nat. Rev. Drug Discov. 15, 327-347 (2016).

2. Vijay, R. \& Perlman, S. Middle East respiratory syndrome and severe acute respiratory syndrome. Curr. Opin. Virol. 16, 70-76 (2016).

3. Ge, X.Y. et al. Isolation and characterization of a bat SARS-like coronavirus that uses the ACE2 receptor. Nature 503, 535-538 (2013).

4. Sabir, J.S. et al. Co-circulation of three camel coronavirus species and recombination of MERS-CoVs in Saudi Arabia. Science 351, 81-84 (2016).

5. Yang, Y. et al. Receptor usage and cell entry of bat coronavirus HKU4 provide insight into bat-to-human transmission of MERS coronavirus. Proc. Natl. Acad. Sci. USA 111, 12516-12521 (2014).

6. Wang, Q. et al. Bat origins of MERS-CoV supported by bat coronavirus HKU4 usage of human receptor CD26. Cell Host Microbe 16, 328-337 (2014).

7. Yang, Y. et al. Two mutations were critical for bat-to-human transmission of Middle East respiratory syndrome coronavirus. J. Virol. 89, 9119-9123 (2015).

8. van der Hoek, L. et al. Identification of a new human coronavirus. Nat. Med. 10, 368-373 (2004).

9. Fouchier, R.A. et al. A previously undescribed coronavirus associated with respiratory disease in humans. Proc. Natl. Acad. Sci. USA 101, 6212-6216 (2004).

10. Hofmann, H. et al. Human coronavirus NL63 employs the severe acute respiratory syndrome coronavirus receptor for cellular entry. Proc. Natl. Acad. Sci. USA 102, 7988-7993 (2005).

11. Chiu, S.S. et al. Human coronavirus NL63 infection and other coronavirus infections in children hospitalized with acute respiratory disease in Hong Kong, China. Clin. Infect. Dis. 40, 1721-1729 (2005).

12. Mole, B. Deadly pig virus slips through US borders. Nature 499, 388 (2013).

13. Du, L. et al. The spike protein of SARS-CoV: a target for vaccine and therapeutic development. Nat. Rev. Microbiol. 7, 226-236 (2009).

14. Bosch, B.J., van der Zee, R., de Haan, C.A. \& Rottier, P.J. The coronavirus spike protein is a class I virus fusion protein: structural and functional characterization of the fusion core complex. J. Virol. 77, 8801-8811 (2003).

15. Burkard, C. et al. Coronavirus cell entry occurs through the endo-/lysosomal pathway in a proteolysis-dependent manner. PLoS Pathog. 10, e1004502 (2014).

16. Millet, J.K. \& Whittaker, G.R. Host cell entry of Middle East respiratory syndrome coronavirus after two-step, furin-mediated activation of the spike protein. Proc. Natl. Acad. Sci. USA 111, 15214-15219 (2014).

17. Millet, J.K. \& Whittaker, G.R. Host cell proteases: critical determinants of coronavirus tropism and pathogenesis. Virus Res. 202, 120-134 (2015).

18. Walls, A.C. et al. Cryo-electron microscopy structure of a coronavirus spike glycoprotein trimer. Nature 531, 114-117 (2016).

19. Li, X. et al. Electron counting and beam-induced motion correction enable nearatomic-resolution single-particle cryo-EM. Nat. Methods 10, 584-590 (2013).

20. Scheres, S.H. \& Chen, S. Prevention of overfitting in cryo-EM structure determination. Nat. Methods 9, 853-854 (2012).

21. Rosenthal, P.B. \& Henderson, R. Optimal determination of particle orientation, absolute hand, and contrast loss in single-particle electron cryomicroscopy. J. Mol. Biol. 333, 721-745 (2003).

22. Brown, A. et al. Tools for macromolecular model building and refinement into electron cryo-microscopy reconstructions. Acta Crystallogr. D Biol. Crystallogr. 71, 136-153 (2015).

23. DiMaio, F. et al. Atomic-accuracy models from $4.5-\AA$ cryo-electron microscopy data with density-guided iterative local refinement. Nat. Methods 12, 361-365 (2015).

24. Wang, R.Y. et al. De novo protein structure determination from near-atomicresolution cryo-EM maps. Nat. Methods 12, 335-338 (2015).

25. Song, Y. et al. High-resolution comparative modeling with RosettaCM. Structure 21, 1735-1742 (2013).

26. Frese, C.K. et al. Unambiguous phosphosite localization using electron-transfer/ higher-energy collision dissociation (EThcD). J. Proteome Res. 12, 1520-1525 (2013).

27. Jeffers, S.A., Hemmila, E.M. \& Holmes, K.V. Human coronavirus $229 \mathrm{E}$ can use CD209L (L-SIGN) to enter cells. Adv. Exp. Med. Biol. 581, 265-269 (2006).

28. Ritchie, G. et al. Identification of $\mathrm{N}$-linked carbohydrates from severe acute respiratory syndrome (SARS) spike glycoprotein. Virology 399, 257-269 (2010).

29. Zhou, Y. et al. A single asparagine-linked glycosylation site of the severe acute respiratory syndrome coronavirus spike glycoprotein facilitates inhibition by mannosebinding lectin through multiple mechanisms. J. Virol. 84, 8753-8764 (2010).
30. Jeffers, S.A. et al. CD209L (L-SIGN) is a receptor for severe acute respiratory syndrome coronavirus. Proc. Natl. Acad. Sci. USA 101, 15748-15753 (2004)

31. Holm, L. \& Rosenström, P. Dali server: conservation mapping in 3D. Nucleic Acids Res. 38, W545-W549 (2010).

32. Kawase, M., Shirato, K., van der Hoek, L., Taguchi, F. \& Matsuyama, S. Simultaneous treatment of human bronchial epithelial cells with serine and cysteine protease inhibitors prevents severe acute respiratory syndrome coronavirus entry. J. Virol. 86, 6537-6545 (2012)

33. Kirchdoerfer, R.N. et al. Pre-fusion structure of a human coronavirus spike protein. Nature 531, 118-121 (2016).

34. McLellan, J.S. et al. Structure of RSV fusion glycoprotein trimer bound to a prefusion-specific neutralizing antibody. Science 340, 1113-1117 (2013).

35. Yin, H.S., Wen, X., Paterson, R.G., Lamb, R.A. \& Jardetzky, T.S. Structure of the parainfluenza virus $5 \mathrm{~F}$ protein in its metastable, prefusion conformation. Nature 439, 38-44 (2006).

36. Harrison, S.C. Viral membrane fusion. Nat. Struct. Mol. Biol. 15, 690-698 (2008).

37. Zheng, Q. et al. Core structure of S2 from the human coronavirus NL63 spike glycoprotein. Biochemistry 45, 15205-15215 (2006)

38. Dormitzer, P.R., Sun, Z.Y., Wagner, G. \& Harrison, S.C. The rhesus rotavirus VP4 sialic acid binding domain has a galectin fold with a novel carbohydrate binding site. EMBO J. 21, 885-897 (2002).

39. Krempl, C., Schultze, B. Laude, H.\& Herrler, G. Point mutations in the S protein connect the sialic acid binding activity with the enteropathogenicity of transmissible gastroenteritis coronavirus. J. Virol. 71, 3285-3287 (1997).

40. Milewska, A. et al. Human coronavirus NL63 utilizes heparan sulfate proteoglycans for attachment to target cells. J. Virol. 88, 13221-13230 (2014).

41. Peng, G. et al. Crystal structure of bovine coronavirus spike protein lectin domain J. Biol. Chem. 287, 41931-41938 (2012)

42. Li, F. Evidence for a common evolutionary origin of coronavirus spike protein receptor-binding subunits. J. Virol. 86, 2856-2858 (2012).

43. Wu, K., Li, W., Peng, G. \& Li, F. Crystal structure of NL63 respiratory coronavirus receptor-binding domain complexed with its human receptor. Proc. Natl. Acad. Sci. USA 106, 19970-19974 (2009).

44. Reguera, J. et al. Structural bases of coronavirus attachment to host aminopeptidase $\mathrm{N}$ and its inhibition by neutralizing antibodies. PLoS Pathog. 8, e1002859 (2012).

45. Ying, T. et al. Junctional and allele-specific residues are critical for MERS-CoV neutralization by an exceptionally potent germline-like antibody. Nat. Commun. 6, 8223 (2015).

46. Prabakaran, P. et al. Structure of severe acute respiratory syndrome coronavirus receptor-binding domain complexed with neutralizing antibody. J. Biol. Chem. 281, 15829-15836 (2006)

47. Hwang, W.C. et al. Structural basis of neutralization by a human anti-severe acute respiratory syndrome spike protein antibody, 80R. J. Biol. Chem. 281 34610-34616 (2006)

48. Sui, J. et al. Potent neutralization of severe acute respiratory syndrome (SARS coronavirus by a human $\mathrm{mAb}$ to $\mathrm{S} 1$ protein that blocks receptor association. Proc. Natl. Acad. Sci. USA 101, 2536-2541 (2004).

49. Zhu, Z. et al. Potent cross-reactive neutralization of SARS coronavirus isolates by human monoclon al antibodies. Proc. Natl. Acad. Sci. USA 104, 12123-12128 (2007)

50. Chen, B. et al. Structure of an unliganded simian immunodeficiency virus gp120 core. Nature 433, 834-841 (2005).

51. Huang, C.C. et al. Structure of a V3-containing HIV-1 gp120 core. Science 310, 1025-1028 (2005)

52. Stewart-Jones, G.B. et al. Trimeric HIV-1-Env structures define glycan shields from clades A, B, and G. Cell 165, 813-826 (2016).

53. Sommerstein, R. et al. Arenavirus glycan shield promotes neutralizing antibody evasion and protracted infection. PLoS Pathog. 11, e1005276 (2015).

54. Falkowska, E., Kajumo, F., Garcia, E., Reinus, J. \& Dragic, T. Hepatitis C virus envelope glycoprotein E2 glycans modulate entry, CD81 binding, and neutralization. J. Virol. 81, 8072-8079 (2007)

55. Szakonyi, G. et al. Structure of the Epstein-Barr virus major envelope glycoprotein. Nat. Struct. Mol. Biol. 13, 996-1001 (2006). 


\section{ONLINE METHODS}

Plasmids. A gene fragment encoding the HCoV-NL63 Sectodomain (residues 161291, UniProt Q6Q1S2) was PCR-amplified from a plasmid containing the fulllength $S$ gene. The PCR product was ligated to a gene fragment encoding a GCN4 trimerization motif (LIKRMKQIEDKIEEIESKQKKIENEIARIKKIK) ${ }^{18,35,56}$, a thrombin-cleavage site (LVPRGSLE), an eight-residue-long Strep-Tag (WSHPQFEK) and a stop codon. Subsequent cloning was performed in the pMT-BiP-V5-His expression vector (Invitrogen) in frame with the Drosophila $\mathrm{BiP}$ secretion signal downstream the metallothionein promoter.

Production of recombinant HCoV-NL63 S ectodomain in Drosophila S2 cells. To generate a stable Drosophila $\mathrm{S} 2$ cell line expressing the recombinant $\mathrm{HCoV}$ NL63 S ectodomain, we used Effectene (Qiagen) and $2 \mu \mathrm{g}$ of plasmid. Puromycin $\mathrm{N}$-acetyltransferase was cotransfected and used as a dominant selectable marker. Stable HCoV-NL63 S-expressing cell lines were selected by addition of $7 \mu \mathrm{g} / \mathrm{ml}$ puromycin (Invivogen) to the culture medium $48 \mathrm{~h}$ after transfection. For largescale production, the cells were cultured in spinner flasks and induced by $5 \mu \mathrm{M}$ of $\mathrm{CdCl}_{2}$ at a density of approximately $10^{7}$ cells $/ \mathrm{mL}$. After one week at $28^{\circ} \mathrm{C}$, clarified cell supernatants were concentrated 40 -fold with Vivaflow tangential

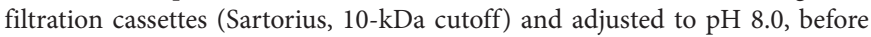
affinity purification with a StrepTactin Superflow column (IBA) followed by gel-filtration chromatography with a Superose 6 10/300 GL column (GE Life Sciences) equilibrated in $20 \mathrm{mM}$ Tris- $\mathrm{HCl}, \mathrm{pH} 7.5$, and $100 \mathrm{mM} \mathrm{NaCl}$. The purified protein was quantified according to absorption at $280 \mathrm{~nm}$ and concentrated to approximately $3 \mathrm{mg} / \mathrm{mL}$.

Cryo-EM specimen preparation and data collection. $2 \mu \mathrm{l}$ of purified $\mathrm{HCoV}$ NL63 spike at $1.0 \mathrm{mg} / \mathrm{mL}$ was applied to a 1.2/1.3 C-flat grid (Protochips), which had been glow-discharged for $30 \mathrm{~s}$ at $20 \mathrm{~mA}$. Grids were then plunge-frozen in liquid ethane with an FEI Mark I Vitrobot with 7.5-s blot time and an offset of $-3 \mathrm{~mm}$ at $100 \%$ humidity and $25^{\circ} \mathrm{C}$. Data were collected with Leginon automatic data-collection software ${ }^{57}$ on an FEI Titan Krios operated at $300 \mathrm{kV}$ and equipped with a Gatan Quantum GIF energy filter, operated in zero-loss mode with a slit width of $20 \mathrm{eV}$, and a Gatan K2 Summit direct electron detector camera. The dose rate was adjusted to 8 counts/pixel/s, and each movie was acquired in counting mode fractionated in 50 frames of $200 \mathrm{~ms}$. 1,400 micrographs were collected in a single session with a defocus range between 2.0 and $4.0 \mu \mathrm{m}$.

Cryo-EM data processing. Whole-frame alignment was carried out with DOSEFGPU DRIFTCORR ${ }^{19}$. The parameters of the microscope contrast-transfer function were initially estimated with CTFFIND4 (ref. 58) and then with GCTF59. Micrographs were manually masked with Appion ${ }^{60}$ to exclude the visible carbon edge from images. Particles were automatically picked with DoGPicker ${ }^{61}$. Particle images were extracted and processed with Relion 1.4 (ref. 62) with a box size of 320 pixels $^{2}$ and a pixel size of $1.36 \AA$. After reference-free $2 \mathrm{D}$ classification, we retained 180,000 out of 474,000 particles to run $3 \mathrm{D}$ classification with C1 symmetry ${ }^{62}$. We used the initial model previously generated for $\mathrm{MHV}^{18}$ with Optimod ${ }^{63}$ and low-pass-filtered the data to $60 \AA$ as a starting reference for 3D classification. 118,000 particles were selected and used to run gold-standard $3 \mathrm{D}$ refinement with Relion ${ }^{20}$, thus yielding a map at $3.95-\AA ̊$ resolution. After particle-motion and radiation-damage correction with Relion particle polishing ${ }^{64}$, another round of 3 D classification with C3 symmetry was performed to select 79,667 particles. After gold-standard 3D refinement with this subset of particles, we obtained a reconstruction at 3.76- $\AA$ resolution. Per-particle defocus parameters were estimated with GCTF and used to run an identical round of 3D refinement that yielded the final 3.4- $\AA$-resolution map. Post processing was performed with Relion to apply an automatically generated $B$ factor of $-129 \AA^{2}$. Reported resolutions were based on the gold-standard FSC $=0.143$ criterion $^{20,21}$, and FSC curves were corrected for the effects of soft masking by high-resolution noise substitution ${ }^{65}$. The soft mask used for FSC calculation had a 10-pixel cosine-edge fall-off.

Model building and analysis. UCSF Chimera ${ }^{66}$ and $\operatorname{Coot}^{22,67}$ were used to fit atomic models into the cryo-EM map. The MHV $\mathrm{S}_{2}$ subunit was fit into the density and rebuilt manually in Coot. The crystal structure of HCoV-NL63 domain $\mathrm{B}$ was then fit into the density, and the rest of the $S_{1}$ subunit was built with a combination of manual building in Coot and de novo building with Rosetta ${ }^{23-25}$. Glycan density coming after an NXS/T motif was initially manually built into the density, and glycan geometry was then refined with Rosetta, optimizing the fit-to-density as well as the energetics of protein-glycan contacts. The glycans were not as well defined as the protein region in the reconstruction, owing to flexibility and compositional heterogeneity. The final model was refined by application of strict noncrystallographic symmetry constraints with Rosetta, with a training map corresponding to one of the two maps generated by the goldstandard refinement procedure in Relion. The second map (testing map) was used only for calculation of the FSC compared with the atomic model and preventing overfitting ${ }^{68}$. The quality of the final model was analyzed with MolProbity ${ }^{69}$ and Privateer ${ }^{70}$. Structure analysis was performed with the DALI server ${ }^{31}$ and areaimol ${ }^{71}$. Electrostatic-potential calculations were performed with PDB2PQR ${ }^{72}$ and $\mathrm{APBS}^{73}$. All figures were generated with UCSF Chimera ${ }^{66}$. Local resolution estimation was performed with Resmap ${ }^{74}$.

Mass spectrometry. HCoV-NL63 S was prepared for MS analysis unaltered or subjected to Endo H (NEB), subjected to Endo F3 (Millipore) or subjected to combined Endo $\mathrm{H}$ and Endo F3 deglycosylation treatment. $2 \mu \mathrm{l}$ of the relevant endoglycosidases was incubated with $20 \mu \mathrm{g}$ of $\mathrm{HCoV}-\mathrm{NL} 63 \mathrm{~S}$ for $14 \mathrm{~h}$ overnight in $50 \mathrm{mM}$ sodium acetate, $\mathrm{pH} 4.4$, at $37^{\circ} \mathrm{C}$ in a $20-\mu \mathrm{L}$ reaction. $6 \mu \mathrm{g}$ of $\mathrm{HCoV}$ NL63 S was then incubated in a freshly prepared solution containing $100 \mathrm{mM}$ Tris, $\mathrm{pH} 8.5,2 \%$ sodium deoxycholate, $10 \mathrm{mM}$ Tris(2-carboxyethyl)phosphine and $40 \mathrm{mM}$ iodoacetamide at $95{ }^{\circ} \mathrm{C}$ for $5 \mathrm{~min}$; this was followed by an incubation at $25^{\circ} \mathrm{C}$ for $30 \mathrm{~min}$ in the dark. $1.6 \mu \mathrm{g}$ of denatured, reduced and alkylated HCoV-NL63 S was then diluted into freshly prepared $50 \mathrm{mM}$ ammonium bicarbonate and incubated for $14 \mathrm{~h}$ at $37^{\circ} \mathrm{C}$ with $0.032 \mu \mathrm{g}$ of either trypsin (Sigma Aldrich) or chymotrypsin (Sigma Aldrich). Formic acid was then added to a final concentration of $2 \%$ to precipitate the sodium deoxycholate in the samples. Samples were then centrifuged at 14,000 r.p.m. for $20 \mathrm{~min}$. The supernatant containing the (glyco)peptides was collected and spun again at 14,000 r.p.m. for $5 \mathrm{~min}$ immediately before sample analysis. Between 4 and $7 \mu \mathrm{L}$ was run on a Thermo Scientific Orbitrap Fusion Tribrid mass spectrometer. A $35-\mathrm{cm}$ analytical column and a 3-cm trap column filled with ReproSil-Pur C18AQ $5 \mu \mathrm{m}$ (Dr. Maisch) beads were used. Nanospray LC-MS/MS was used to separate peptides over a 110 -min gradient from $5 \%$ to $30 \%$ acetonitrile with $0.1 \%$ formic acid. A positive spray voltage of 2,100 was used with an ion-transfer-tube temperature of $350{ }^{\circ} \mathrm{C}$. An electron-transfer/higher-energy collision dissociation ion-fragmentation scheme ${ }^{26}$ was used with calibrated charge-dependent ETD parameters and a supplemental higher-energy collision dissociation energy of 0.15 for the samples with intact glycopeptides and 0.2 for the samples treated with endoglycosidases. A resolution setting of 120,000 with an AGC target of $2 \times 10^{5}$ was used for MS1, and a resolution setting of 30,000 with an AGC target of $1 \times 10^{5}$ was used for MS2. The data were searched against a custom database including recombinant coronavirus S-glycoprotein sequences, a list of common contaminant proteins including trypsin, chymotrypsin and the endoglycosidases, as well as 998 decoy reverse yeast sequences, with trypsin or chymotrypsin as the protease, allowing up to two missed cleavages. All searches included carbamidomethylation of cysteine as a fixed modification and oxidation of methionine as a variable modification. An initial comprehensive search for glycosylation revealed that (core-fucosylated) paucimannose and high-mannose structures were the only identified glycan species in the samples. On the basis of these findings, a final search was performed with $\mathrm{COMET}^{75}$ on the same data with the following list of variable modifications of asparagine residues: $+\mathrm{HexNAc}(2) \mathrm{Hex}(3),+\mathrm{HexNAc}(2) \mathrm{Hex}(3) \mathrm{dHex}(1)$, $+\operatorname{HexNAc}(2) \mathrm{Hex}(3) \mathrm{dHex}(2),+\operatorname{HexNAc}(2) \mathrm{Hex}(4),+\operatorname{HexNAc}(2) \mathrm{Hex}(5)$, $+\operatorname{HexNAc}(2) \operatorname{Hex}(6), \quad+\operatorname{HexNAc}(2) \operatorname{Hex}(7), \quad+\operatorname{HexNAc}(2) \mathrm{Hex}(8) \quad$ and $+\operatorname{HexNAc}(2) \mathrm{Hex}(9)$. The samples treated with endoglycosidases were searched with $+\operatorname{HexNAc},+\operatorname{HexNAc}(1) \mathrm{dHex}(1)$ and $+\operatorname{HexNAc}(1) \mathrm{dHex}(2)$ as variable modifications of asparagine. We used a precursor mass tolerance of 20 p.p.m., 0.02 fragment bin size, including $\mathrm{b} / \mathrm{c} / \mathrm{y} / \mathrm{z}$ fragments, with monoisotopic masses for both precursor and fragment ions. The search results were filtered for modification of asparagine residues and the presence of an NX(S/T) sequon at the protein level. All appropriate peptide spectrum matches (PSMs) were manually inspected, and only those with reasonable peptide sequence coverage were kept. In addition, the spectra were inspected for the presence of glycan fragment ions. All glycosylation sites identified by MS listed in Supplementary Table 1 are based on multiple PSMs, often with multiple different glycans and additional confirmation from overlap between the trypsin- and 
chymotrypsin-treated samples. The greatest number of glycopeptide identifications was made in the chymotrypsin-digested samples.

Hemagglutination assay. The $\mathrm{S}_{1}$ subunit of HCoV-NL63 C-terminally tagged with the $\mathrm{Fc}$ portion of human $\operatorname{IgG}\left(\mathrm{S}_{1}-\mathrm{Fc}\right)$ was tested alone or premixed with $1 \mu \mathrm{l}$ of Protein A-coupled, 200-nm-sized nanoparticles (nano-screenMAG-Protein A beads; Chemicell, cat.no. 4503-1) to increase the avidity of $S_{1}$-Fc proteins for sialic acids on the erythrocyte surface. The sialic acid-binding $S_{1}$ subunit of PEDV (strain GDU, GenBank AFP81695.1) C-terminally fused to the human Fc portion was used as a positive control. 'Mock' indicates the conditions in which no $S_{1}$ subunit was used (negative control). The initial concentration of $\mathrm{S}_{1}$-Fc was $5 \mu \mathrm{g}$, and two-fold serial dilutions of $\mathrm{S}_{1}$-Fc-nanoparticle mixtures were made in $50 \mu \mathrm{l}$ phosphate-buffered saline supplemented with $0.1 \%$ bovine serum albumin. $50 \mu \mathrm{l}$ erythrocyte suspension $(0.5 \%)$ was mixed with $50 \mu \mathrm{l}$ of $\mathrm{S}_{1}$-Fc-nanoparticle dilution in $\mathrm{V}$-shaped 96-well plates and incubated for $2 \mathrm{~h}$ on ice, after which the wells were photographed.

Protein expression of $S_{1}$ variants and ACE2. Different $S_{1}$ variants of HCoVNL63 $S$ protein, including $S_{1}$ (residues 1-718), $S_{1}$ domain 0 ( $S_{1}-0$, residues 1-209) and $S_{1}$ domain $B\left(S_{1}-B\right.$, residues 481-616), were $C$-terminally fused to the $F c$ region of mouse $\operatorname{IgG}(\mathrm{mFc})$, expressed in HEK-293T cells and affinity purified as previously described ${ }^{76}$. Likewise, an $\mathrm{S}_{1}-\mathrm{mFc}$ expression plasmid was made for the SARS-CoV $S_{1}$ domain (isolate CUHK-W1, residues 1-676) and the PEDV $\mathrm{S}_{1}$ domain (strain GDU; residues 1-728). Expression of the human angiotensinconverting enzyme ectodomain (ACE2; residues 1-614) fused to the Fc portion of human $\operatorname{IgG}(\mathrm{hFc})$ was performed as previously described ${ }^{76}$.

ACE2 binding ELISA. The ability of the HCoV-NL63 $\mathrm{S}_{1}-\mathrm{mFc}$ and $\mathrm{S}_{1}-\mathrm{B}-\mathrm{mFc}$ chimeric proteins to bind the ACE2-hFc receptor was evaluated with an ELISA-based assay. $100 \mu \mathrm{l}$ of hACE2-hFc $(20 \mu \mathrm{g} / \mathrm{ml}$, diluted in PBS $)$ was coated on a 96-well MaxiSorb plate overnight at $4{ }^{\circ} \mathrm{C}$. Nonspecific binding sites were subsequently blocked with a $3 \%(\mathrm{w} / \mathrm{v})$ solution of bovine serum albumin in PBS. Plates were washed with washing buffer (PBS with $0.05 \%$ Tween 20 ) and subsequently incubated with serially diluted $\mathrm{S}_{1}-\mathrm{mFc}$ proteins (starting with equimolar concentrations) for $1 \mathrm{~h}$ at room temperature, after which plates were washed three times with washing buffer. $\mathrm{mFc}$-tagged $\mathrm{S}_{1}$ proteins were detected with $\mathrm{HRP}$-conjugated polyclonal rabbit-anti-mouse immunoglobulins (1:2,000 dilution in PBS with $0.1 \%$ BSA; DAKO, P0260; validation on manufacturer's website), and a colorimetric reaction was produced after incubation with tetramethylbenzidine substrate (BioFX). The optical density (OD) was subsequently measured at $450 \mathrm{~nm}$ with an ELISA reader (EL-808, BioTEK). Background (signal from HRP-conjugated anti$\mathrm{mFc}$ antibody alone) was subtracted from the $\mathrm{OD}_{450 \mathrm{~nm}}$ values. The $\mathrm{mFc}$-tagged SARS-CoV $\mathrm{S}_{1}$ subunit was used as a positive control, whereas the $\mathrm{mFc}$-tagged HCoV-NL63 $\mathrm{S}_{1}$ domain 0 (HCoV-NL63 $\mathrm{S}_{1}-0-\mathrm{mFc}$ ) and PEDV $\mathrm{S}_{1}$ subunit (PEDV $\mathrm{S}_{1}-\mathrm{mFc}$ ), both of which do not bind ACE2, were used as negative controls.

Surface plasmon resonance (SPR). SPR was performed on a GE Healthcare Biacore T200 with a running buffer containing $20 \mathrm{mM}$ HEPES, pH 7.5, $100 \mathrm{mM}$ $\mathrm{NaCl}$ and $0.5 \%$ Tween- 20 , with a flow rate of $30 \mu \mathrm{L} / \mathrm{min}$ at $25^{\circ} \mathrm{C}$. A carboxymethylated dextran (CM5) chip (GE Healthcare) was activated with $N$-hydroxysulfosuccinimide (NHS) and 1-ethyl-3-(3-dimethylaminopropyl) carbodiimide
(EDC). We then either quenched the CM5 surface with ethanolamine (yielding a blank flow cell) or immobilized HCoV-NL63 S before quenching. $10 \mu \mathrm{g}$ of HCoV-NL63 S was diluted into $10 \mathrm{mM}$ sodium acetate, $\mathrm{pH} 5.5$ and was directly immobilized for $700 \mathrm{~s}$, thus yielding 28,000 RUs. After immobilization quenching, running buffer was flowed for $10 \mathrm{~min}$ to ensure a steady baseline before experimental binding. Heparan sulfate (Sigma Aldrich) was reconstituted in running buffer at $5.0 \mathrm{mg} / \mathrm{mL}$. Two concentrations of heparan sulfate, $5.0 \mathrm{mg} / \mathrm{mL}$ and $2.5 \mathrm{mg} / \mathrm{mL}$, were injected for $80 \mathrm{~s}$ with a dissociation time of $400 \mathrm{~s}$. All data were subtracted from the blank flow cell, to account for any nonspecific interactions of heparan sulfate with the CM5 chip, and the baseline was normalized to 0 .

56. Eckert, D.M., Malashkevich, V.N. \& Kim, P.S. Crystal structure of GCN4-pIQI, a trimeric coiled coil with buried polar residues. J. Mol. Biol. 284, 859-865 (1998).

57. Suloway, C. et al. Automated molecular microscopy: the new Leginon system. J. Struct. Biol. 151, 41-60 (2005).

58. Rohou, A. \& Grigorieff, N. CTFFIND4: fast and accurate defocus estimation from electron micrographs. J. Struct. Biol. 192, 216-221 (2015).

59. Zhang, K. Gctf: Real-time CTF determination and correction. J. Struct. Biol. 193, 1-12 (2016).

60. Lander, G.C. et al. Appion: an integrated, database-driven pipeline to facilitate EM image processing. J. Struct. Biol. 166, 95-102 (2009).

61. Voss, N.R., Yoshioka, C.K., Radermacher, M., Potter, C.S. \& Carragher, B. DoG Picker and TiltPicker: software tools to facilitate particle selection in single particle electron microscopy. J. Struct. Biol. 166, 205-213 (2009).

62. Scheres, S.H. RELION: implementation of a Bayesian approach to cryo-EM structure determination. J. Struct. Biol. 180, 519-530 (2012).

63. Lyumkis, D., Vinterbo, S., Potter, C.S. \& Carragher, B. Optimod: an automated approach for constructing and optimizing initial models for single-particle electron microscopy. J. Struct. Biol. 184, 417-426 (2013).

64. Scheres, S.H. Beam-induced motion correction for sub-megadalton cryo-EM particles. eLife 3, e03665 (2014).

65. Chen, S. et al. High-resolution noise substitution to measure overfitting and validate resolution in $3 \mathrm{D}$ structure determination by single particle electron cryomicroscopy. Ultramicroscopy 135, 24-35 (2013).

66. Goddard, T.D., Huang, C.C. \& Ferrin, T.E. Visualizing density maps with UCSF Chimera. J. Struct. Biol. 157, 281-287 (2007).

67. Emsley, P., Lohkamp, B., Scott, W.G. \& Cowtan, K. Features and development of Coot. Acta Crystallogr. D Biol. Crystallogr. 66, 486-501 (2010).

68. DiMaio, F., Zhang, J., Chiu, W. \& Baker, D. Cryo-EM model validation using independent map reconstructions. Protein Sci. 22, 865-868 (2013).

69. Chen, V.B. et al. MolProbity: all-atom structure validation for macromolecular crystallography. Acta Crystallogr. D Biol. Crystallogr. 66, 12-21 (2010).

70. Agirre, J. et al. Privateer: software for the conformational validation of carbohydrate structures. Nat. Struct. Mol. Biol. 22, 833-834 (2015).

71. Lee, B. \& Richards, F.M. The interpretation of protein structures: estimation of static accessibility. J. Mol. Biol. 55, 379-400 (1971).

72. Dolinsky, T.J., Nielsen, J.E., McCammon, J.A. \& Baker, N.A. PDB2PQR: an automated pipeline for the setup of Poisson-Boltzmann electrostatics calculations. Nucleic Acids Res. 32, W665-W667 (2004).

73. Baker, N.A., Sept, D., Joseph, S., Holst, M.J. \& McCammon, J.A. Electrostatics of nanosystems: application to microtubules and the ribosome. Proc. Natl. Acad. Sci. USA 98, 10037-10041 (2001).

74. Kucukelbir, A., Sigworth, F.J. \& Tagare, H.D. Quantifying the local resolution of cryo-EM density maps. Nat. Methods 11, 63-65 (2014).

75. Eng, J.K., Jahan, T.A. \& Hoopmann, M.R. Comet: an open-source MS/MS sequence database search tool. Proteomics 13, 22-24 (2013).

76. Raj, V.S. et al. Dipeptidyl peptidase 4 is a functional receptor for the emerging human coronavirus-EMC. Nature 495, 251-254 (2013) 